\title{
Autoimmune diseases, their pharmacological treatment and the cardiovascular system
}

\author{
Marta Jastrzębska ${ }^{1}$, Michael E. Czok ${ }^{2}$, Przemysław Guzik ${ }^{1,2}$ \\ ${ }^{1}$ Department of Cardiology and Intensive Therapy, Heliodor Swiecicki University Hospital, Poznan, Poland \\ ${ }^{2}$ Department of Cardiology and Intensive Therapy, Poznan University of Medical Sciences, Poznan, Poland
}

\begin{abstract}
Cardiovascular system involvement is a frequent complication of autoimmune diseases $(A D)$ such as systemic lupus erythematosus, scleroderma, rheumatoid arthritis, spondyloarthropaties or psoriatic arthritis. The most common forms of such involvement are pericarditis, myocarditis, accelerated atherosclerosis resulting in myocardial infarction or stroke, arrhythmias, conduction abnormalities or congestive heart failure. Some of these manifestations may be dramatic in their course and ultimately fatal. The treatment of $A D$ may further affect the cardiovascular system and result in a lower quality of life, higher mortality and increased cost of healthcare. The aim of this review is to discuss possible cardiac complications of various $A D$ and the related treatment of these diseases. (Cardiol J 2013; 20, 6: 569-576)
\end{abstract}

Key words: cardiovascular complications, autoimmune diseases, heart failure, adverse drug effects

\section{Introduction}

In developed countries approximately $25 \%$ of the general population suffers from some form of autoimmune disorders [1]. Autoimmune diseases (AD) are characterized by a primary dysfunction of the immune system with increased level of autoantibodies, inflammatory and mediatory cells resulting in chronic inflammation of connective tissue [2]. The risk factors influencing the development of $\mathrm{AD}$ include: advancing age, gender, genetics, environmental insults, and infections [3]. Women tend to be stricken more often with rheumatoid arthritis, systemic lupus erythematosus (SLE) and scleroderma (SCL), while men tend to suffer from ankylosing spondylitis and certain types of vasculitis.

$\mathrm{AD}$ can be subdivided into organ specific and non-organ-specific [2]. The examples of organ specific $\mathrm{AD}$ include type 1 diabetes mellitus, which results from pancreatic insufficiency following the annihilation of beta cells through the invasion of T-cells, leading to insulin deficiency; Hashimoto's thyroiditis and Graves' disease affecting the thyroid gland but resulting in multi-system effect. Diffuse non-organ specific autoimmune diseases, such as SLE or SCL, tend to have a multi system involvement, including the cardiovascular (CV) system.

\section{Autoimmune diseases and the cardiovascular system}

Pericarditis is the most common CV complication of $\mathrm{AD}$ and is considered to be immune-mediated, although co-existing infection may play a role in some cases [1]. It is a frequent symptom in SCL (50\% of patients), SLE and rheumatoid arthritis (30\% each), and its extension generally reflects the activity of the systemic involvement of $\mathrm{AD}$ [2]. Asymptomatic pericardial effusion and/or

Address for correspondence: Przemyslaw Guzik, MD, PhD, ISHNE Fellow, FESC, Associate Professor of Medicine, Department of Cardiology-Intensive Therapy, Poznan University of Medical Sciences, ul. Przybyszewskiego 49, 60-355 Poznań, Poland, tel: +48 6186916 38, fax: +48 6186916 89, e-mail: pguzik@ptkardio.pl

Received: 27.01.2013 Accepted: 25.04.2013 
pericarditis may be the primary and sole manifestations of an underlying autoimmune process. Arrhythmias or conduction disorders are quite frequent in $\mathrm{AD}$. They tend to be caused by an infiltration of inflammatory cells with ensuing fibrosis of the conductive system and myocardium. Inflammatory infiltrates are usually observed in SCL, systemic lupus erythematosus, rheumatoid arthritis, and seldom in polymyositis, dermatomyositis and mixed connective tissue disease. Autoimmune mediated inflammatory process may also affect coronary arteries and lead towards ischemic heart disease or acute coronary syndromes. Non-infective endocarditis with primary involvement of the left heart valves is observed in systemic lupus erythematosus. Pulmonary hypertension, developing during the course of $\mathrm{AD}$ is an example of an indirect effect on cardiac function. Autoimmune inflammatory processes can have disastrous effects on major vessels, causing aneurysms, arterial and venous thrombi and various forms of embolization. These can develop into emergent and potentially fatal scenarios. It would be worthy to note that each of ADs directly or indirectly affects the $\mathrm{CV}$ system with varying severity [2-4].

SLE is an inflammatory disease of the connective tissue, affecting approximately $0.1 \%$ of the general population, with the female-to-male ratio $6-10: 1$. Pericarditis manifests in just a bit over $50 \%$ of SLE patients, however they may remain asymptomatic. In certain cases pericarditis may precede clinical signs of SLE or exacerbate the underlying disease. On CV imaging, small pericardial effusions and thickening of pericardial plaques may be observed in SLE patients. Clinically urgent pericarditis occurs in $30 \%$ of patients, with substantial fluid accumulation resulting in cardiac tamponade $[5,6]$. In the latest clinical evaluations, cardiac tamponade was found in $22 \%$ of patients, whose management required high-dose corticosteroids and in half these cases - a pericardial window [7]. Antiphospholipid antibodies, detected in approximately $30-50 \%$ of SLE patients, are the main risk factor for CV complications [6]. These antibodies cause complement activation and deposition of immune complexes in tissues and may affect the conductive system of the heart. Non-infective verrucous vegetations found on valves, first described in 1924 as the Libman-Sachs endocarditis, are one of the most characteristic symptoms of SLE. In $63 \%$ of cases the mitral valve is involved, however aortic valve or multivalvular involvement is relatively common. Libman-Sachs endocarditis occurs in $50 \%$ of patients with SLE [8].
It is usually mild but in approximately $20 \%$ of cases have a severe progression to valvular dysfunction requiring surgical intervention [9-15]. Over the natural course of SLE, several inflammatory changes take place within the endothelium (accumulation of inflammatory cells, thickening of intima-media, formation of plaques), leading to premature and accelerated atherosclerosis, with increased risk of coronary heart disease and/or stroke. The risk of acute coronary syndromes is significantly increased, when compared to the general population, in each age group of patients with concomitant SLE and antiphospholipid syndrome, but is particularly greater in young women whose risk is increased 50 -fold. In the John Hopkins SLE Cohort Study, coronary artery disease occurred in over $8 \%$ of the studied patients and was responsible for $30 \%$ of deaths in the 3-year follow-up. In the Toronto Cohort Study $11 \%$ of SLE patients developed coronary artery disease due to the vascular changes. Pulmonary arterial hypertension can occur as patients develop interstitial lung disease or intimal proliferation of pulmonary arteries [16]. Within this patient group, the CV risk is significant and associated with a worse prognosis. Neonatal lupus, a congenital form of SLE, is a passively transferred autoimmune disease. It concerns $1.6-2 \%$ of neonates born to mothers with SLE. It may be diagnosed in young infants causing complete heart block (detected by fetal echocardiography or magnetocardiography at about 20 weeks gestation). Fortunately, neonatal lupus typically resolves by the age of 6 -months $[2,17]$.

In the 8-year follow-up multi-centre study (Atherosclerotic Vascular Events Cohort of SLE), the prevalence of atherosclerotic vascular events was found to vary between $8-13 \%$ within SLE patients, with a peak incidence 8 years after diagnosis. In the Pittsburgh Cohort, the CV risk among women with SLE was found to be approximately $7 \%$. Prolong duration of SLE and extended use of corticosteroid were the main risk factors for the development of CV events [18].

Antiphospholipid syndrome, also known as Hughes syndrome, is an inflammatory disorder that presents with antiphospholipid antibodies causing arterial and venous thrombosis. Pulmonary hypertension is relatively common, occurring in approximately $70 \%$ of patients with antiphospholipid antibodies [19]. Antiphospholipid antibodies cause an activation of complement, followed by deposit formation of immune complexes in tissues. These patients may suffer from angina pectoris and, in some (8\%) advanced cases, myocardial 
infarction (MI). Additionally, there is an increased risk of re-occlusion following coronary angioplasty or coronary artery bypass grafting [20-23].

Valvular (mitral and aortic) regurgitation $(12 \%)$, secondary to their thickening $(30 \%)$ or destruction, is usually a mild form of valvular impairment, but in 4-6\% of patients it progresses to severe valvular defects necessitating surgical intervention. Most of the valvular involvement is clinically silent, some of patients, may have severe thromboembolic events, including stroke [24-26].

SCL is a disease which causes the fibrosis of the skin and internal organs, affecting 70,000 Americans. Female-to-male ratio is 7-10:1 [2]. CV complications in SCL patients include: pericarditis, cardiac arrhythmias, congestive heart failure (HF) and pulmonary hypertension - the main cause of death in SCL patients. In large cohort studies on $\mathrm{SCL}$, the CV system involvement was responsible for up to $36 \%$ of all deaths, while cardiopulmonary deaths accounted for $70 \%$ of the mortality [27]. Symptomatic pericarditis occurs in approximately $20 \%$ of SCL patients, with evidence of pericardial involvement found in $80 \%$ of autopsy cases [28]. Inflammation and autoimmunity stimulate fibroblasts activation, myofibroblasts recruitment, and production of collagen leading to fibrosis. Progression of myocardial fibrosis in SCL results in the development of cardiomyopathy followed by diastolic and systolic HF with significantly reduced left ventricular ejection fraction [29]. Recent studies have revealed myocardial fibrosis in 37\% of SCL autopsy cases. Fibrosis leads to reduced coronary blood flow resulting in myocardial ischemia and may even precipitate MI. In the absence of significant coronary narrowing, a reduction in coronary blood flow reserve is observed in 55\% of asymptomatic patients. This suggests that myocardial involvement is due to the impairment of microcirculation and is associated with structural abnormalities of small coronary vessels [30]. Coronary angiography revealed tortuosity (50\%), calcification $(33 \%)$, stenosis $(20 \%)$, ectasia and slow flow $(20 \%)$, and spasm $(<10 \%)$ in SCL patients [31]. Pulmonary hypertension has a yearly incidence of 6 in 1000 SCL patients as a result of pulmonary vascular obliteration, interstitial fibrosis of pulmonary tissue or left ventricular HF, and less frequently by pulmonary embolization. Lung disease is found in $80 \%$ of SCL patients. A recent meta-analysis of more than 3.5 thousands patients showed that SCL associated pulmonary arterial hypertension (PAH) is particularly aggressive. Untreated PAH is associated with a median survival of 1 year following its diagnosis in SCL patients. PAH remains the main cause of death among SCL patients [31]. The 3-year mortality of SCL patients with PAH varies between $36 \%$ and $53 \%$ with different reports. Mortality is significantly higher $(61-72 \%)$ in SCL patients with pulmonary hypertension secondary to interstitial lung disease [32, 33].

Echocardiography reveals left ventricular systolic dysfunction in about $30 \%$ of patients, and thickening of the aortic and mitral valve leaflets in $12 \%$ and $8 \%$, respectively [2, 34, 35]. A quarter of patients with progressive SCL have developed antibodies to cardiac conducting tissue. ECG studies show that arrhythmias and conduction abnormalities are quite frequent in SCL. These are usually incomplete right bundle branch block $(41 \%)$, benign supraventricular arrhythmias, transient atrial fibrillation, atrial flutter, first degree atrioventricular block, and nodal rhythms (each 10\%). These ECG abnormalities are likely to be transient and result from fibrosis of the myocardium and conductive system. In 6-10\% of SCL patients, sudden cardiac death secondary to ventricular arrhythmia is reported. Studies found that $75 \%$ of SCL patients have a normal resting ECG and 24-h Holter monitoring [34]. Other clinical studies revealed the presence of ventricular arrhythmia in a form of frequent single premature ventricular beats, as bigeminy, trigeminy or pairs in $67 \%$ of SCL patients. More serious ventricular arrhythmias such as non-sustained ventricular tachycardia is observed in 7-13\% of SCL patients. Supraventricular arrhythmias were less frequent in $\mathrm{SCL}$ - atrial fibrillation/flutter or supraventricular paroxysmal tachycardia were found in $20-30 \%$ of patients. Conduction disturbances were diagnosed in $25-75 \%$ of SCL patients. Sudden cardiac deaths were reported in $5-21 \%$ of individuals, usually in patients with skeletal muscle involvement. One effective treatment is cardiac ablation for drug-intolerant and drug-resistant arrhythmias; it, however, should be considered as an alternative treatment. Life-threatening ventricular arrhythmias require an implantable cardioverter defibrillator, whereas advanced conduction disorders need to be managed with a pacemaker implantation [27]. The question must be asked whether it would be warranted to implant a cardioverter-defibrillator device in SCL patients, as a primary prevention.

Rheumatoid arthritis (RA) is an inflammatory disease with distinctive joint malformations affecting about $1-2 \%$ of the general population. Peak incidence occurs in the fourth and fifth decade of 
life, 3 times more often in women. Patients with RA have a significantly increased risk of developing HF (34\%) compared to the general population. In particular, this trend is strongly marked in women, and is constantly higher in the long-term follow-up suggesting that prolonged exposure to inflammatory agents has no impact on the development of HF [36-38]. Similarly, there is a high prevalence of coronary heart disease, with $43 \%$ higher risk for patients with positive rheumatoid factor and as much as 2.5-fold increased risk for patients without the presence of rheumatoid factor. Effects of chronic inflammation, such as endothelial dysfunction and dyslipidemia, increase risk of CV mortality. Silent course of angina pectoris with frequent complication of sudden cardiac death is characteristic for RA. Inflammatory changes, especially in the left anterior descending and left circumflex arteries, are found at autopsies [39-43]. Pericarditis is a common symptom of heart involvement in RA and it is present in $75 \%$ of patients [2]. Constrictive pericarditis may occur in few percent. Pulmonary hypertension is rare and usually secondary to intestinal lung disease. Pathologic examinations revealed inflammatory pulmonary vasculitis, plexiform vasculopathy with intimal hyperplasia and smooth muscle hypertrophy [44]. Pulmonary hypertension may also originate from chronic venous thromboembolic disease, especially in patients with coexisting SCL. Some clinical studies reported an increase in the carotid artery intima-media thickness in RA patients compared to the general population [3]. Certain RA patients may develop valvular dysfunction [45]. CV complications affect $53 \%$ of RA patients [46, 47].

In a retrospective population-based cohort from the Rochester Epidemiology Project, the 10 -year CV risk in RA patients was found to be comparable to that of healthy individuals who were on the average 10 years older [48].

Patients suffering from polymyositis and dermatomyositis, diseases involving the skin and muscles which have co-existing CV disease, are deemed to have a poor prognosis. It is estimated that up to $20 \%$ of patients with polymyositis die from cardiac disease. The average risk of post MI mortality in polymyositis and dermatomyositis is 16 -fold increased; men 9-fold, women up to 32 -fold. Congestive HF (up to $45 \%$ ), angina pectoris (44\%), MI, Prinzmetal angina and pericarditis $(10 \%$ respectively) are the most common manifestation of cardiac involvement in these patients. The frequency of these $\mathrm{CV}$ complications varies depending on the population selected and diagnostic methods used. Another reported CV complications are arrhythmias and conductive abnormalities, which include supraventricular or ventricular tachycardia, atrioventricular blocks, bundle branch blocks, nonspecific ST-T changes and abnormal $\mathrm{Q}$ waves occurring in $32 \%$ of patients [49]. Lymphocyte infiltration throughout the sinoatrial node is rare, however when it occurs, it results in a complete heart block requiring a pacemaker implantation. Extensive deposits of inflammatory cells (primarily $\mathrm{T}$ lymphocytes and macrophages) followed by degeneration of cardiac myocytes and secondary restrictive cardiomyopathy. As a result, severe congestive HF with significantly reduced left ventricular ejection fraction is observed. Interstitial lung disease is also found in 5-30\% of patients and leads to right ventricular HF [49].

The Quebec cohort study recruited 607 patients with dermatomyositis and polymyositis showed the risk of MI equal to 5.3/1000 person-years. Higher incidence of MI in patients with dermatomyositis and polymyositis comparing to the general population was observed in all demographic groups except men younger than 65 years. In this cohort, the rate of stroke was 5.1/1000 person-years, twice as high as in healthy subjects (3.1/1000 person-years) and similar to recorded in RA patients.

In the case-control study of the Healthcare Cost and Utilization Project, data from over 50,000 hospitalizations of patients with dermatomyositis (20\% of US hospitals) were analyzed. For dermatomyositis patients, the rate of death and CV diseases was 2-fold higher than in age- and gender-matched patients without dermatomyositis. Congestive $\mathrm{HF}$ was one of the most frequent causes of death in these patients. This study also found that the prevalence of hypertension and diabetes mellitus among dermatomyositis patients was significantly higher than in the general population $(62 \%$ and $29 \%$ compared with $9 \%$ and $4 \%$, respectively). Similar findings were observed in patients with dermatomyositis or polymyositis regarding any cerebrovascular accident [50].

Spondyloarthropaties are inflammatory diseases of the vertebral column. The most common of these - ankylosing spondylitis (AS) is diagnosed in approximately $1.5 \%$ of the population, with 2-3:1 male-to-female ratio, affecting younger male population. The most common CV complications found in patients with AS are: aortic regurgitation (up to $50 \%$ ), inflammation of the ascending aorta, atrio-ventricular blocks, bundle branch blocks and pericarditis. Presence of the HLA-B27 gene 
predisposes individuals to proximal aortitis, diastolic dysfunction and conduction abnormalities [51]. Aortic root disease is reported in $100 \%$ of autopsies and in over $80 \%$ of transoesophageal echocardiographies. Twenty percent of patients with ascending aorta disease may have either congestive HF, experienced stroke or undergone valve replacement surgery [52].

Psoriatic arthritis affects about $0.5 \%$ of the general population. The inflammation of the ascending aorta with secondary aortic valve involvement may develop as a result of psoriatic arthritis. This is, however, an extremely rare complication. $\mathrm{CV}$ symptoms are significantly more frequent in patients with reactive arthritis. Pericarditis, myocarditis, inflammation of initial segment of the ascending aorta with secondary aortic valve regurgitation, and nonspecific ST-T changes or conduction abnormalities are diagnosed [53].

Summary of the CV complications in autoimmune diseases is shown in Table 1.

\section{Treatment of autoimmune diseases and cardiovascular system}

In spite of the unfavorable influence of autoimmune diseases, $\mathrm{AD}$ treatment has also a significant impact on the CV system. The pharmaceutical treatment of autoimmune inflammatory diseases takes a toll on the CV system, creating a broad array of side effects. The most common drugs used in $\mathrm{AD}$ pharmacotherapy are non-steroidal anti-inflammatory drugs (NSAIDs), glucocorticoids, and so-called disease-modifying antirheumatic drugs (DMARDs) like methotrexate, sulfasalazine or chloroquine. Cyclophosphamide, cyclosporine, and biological treatment are also used.

Glucocorticoids are associated with hypertension $(62 \%)$, diabetes $(18 \%)$, dyslipidemia $(66 \%)$ or thromboembolic complications. They alter kidney function inducing fluid and electrolyte disorders, resulting in congestive HF and hypertension [54, 55]. Glucocorticoids are generally considered to increase the $\mathrm{CV}$ risk. Although, the case-control nationwide study has not confirmed this finding [50]. Contrary, results from the QUEST-RA study showed that glucocorticoids use is accompanied by a small decrease in risk of all CV events [56]. Recent studies in SLE and RA have suggested that corticosteroids have a potential to reduce $\mathrm{CV}$ risk probably by lowering the autoimmune activity and the severity of inflammation [57].

NSAIDs therapy increases the risk of MI, cerebrovascular accident, congestive HF and systemic hypertension [58]. NSAIDs are especially

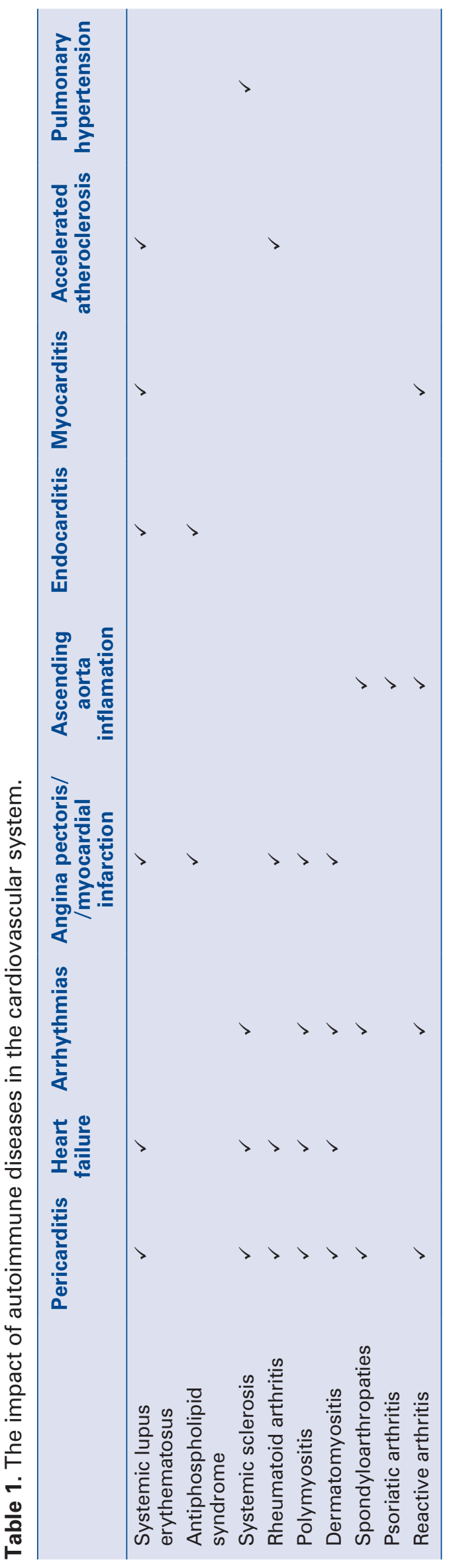


dangerous in the elderly, as they may exacerbate pre-existing HF due to renal dysfunction [59, 60]. The cyclooxygenase-2 (COX-2) inhibitors may create a pro-thrombotic/coagulation imbalance on the endothelial surface, with thrombus formation and potentially fatal embolization. Two large meta-analyses based on over 90 clinical trials showed that NSAIDs increase blood pressure, with higher elevations in hypertensive patients. This drug-induced elevation of blood pressure is associated with a reduction in blood concentrations of prostaglandins and renin. Among the NSAIDs reviewed, piroxicam induces the highest increase in blood pressure, whereas aspirin and flurbiprofen evoke a lower increase in overall blood pressure. NSAIDs interfere with the anti-hypertensive effects of several classes of CV drugs, including: diuretics, angiotensin-converting enzyme inhibitors and beta-blockers. Sustained use of non-selective NSAIDs and selective COX-2 inhibitors results in an extremely increased hazard ratio for ischemic stroke, 1.7 and 4.5, respectively. Furthermore, NSAIDs therapy is associated with sodium and water retention, with double the hospitalizations due to congestive HF [58-60].

Patients being treated with sulfasalazine and leflunomid may develop pericarditis and hypertension, respectively. Chloroquine an antimalarial drug, which has shown a great benefit in the management of ADs has been associated with widening of the QRS complex, QT interval prolongation, $\mathrm{T}$ wave changes and in rare cases complete heart block, necessitating pacemaker implantation [61-63]. Cardiomyopathy and ensuing congestive $\mathrm{HF}$ resulting from long-term chloroquine therapy have also been reported, albeit rarely [64-66]. In the case-control study, DMARDs (especially azathioprine) correlate inversely with the $\mathrm{CV}$ events in dermatomyositis and polymyositis. Hydroxychloroquine improved lipid profiles in SLE patients, however this study failed to prove its beneficial effect on the clinical events form the $\mathrm{CV}$ system [50]. A huge QUEST-RA study (2005-2006) recruiting over 4000 patients from 15 countries proved that one year treatment with methotrexate was associated with $15 \%, 18 \%$, and $11 \%$ decreases of risk for all CV events, MI, and stroke, respectively [56]. Methotrexate may cause pericarditis or pericardial effusions, and lead to pulmonary fibrosis and congestive HF. Methotrexate may increase the risk of hypotension, diabetes and thromboembolic events (arterial thrombosis, cerebral thrombosis, deep vein thrombosis, retinal vein thrombosis, pulmonary embolization). However, methotrexate has been of some benefit to the $\mathrm{CV}$ system as it has been reported to reduce atherosclerotic lesions in coronary arteries [67]. It also can be used as adjunctive therapy for asthma to reduce inflammation.

Infliximab increases the frequency of arrhythmias, hypertension and $\mathrm{HF}$, and in rare cases may cause episodes of hypotension. However, the large population QUEST-RA study revealed lowered risk for all CV events in patients with a long history of TNF-alpha blockers treatment [56].

Etanercept, an immunosuppressive agent with an extremely long half-life of $102 \pm 30 \mathrm{~h}$, is used to treat rheumatoid arthritis. Etanercept has many side effects from increased risk of coronary artery disease, MI, chronic HF, deep vein thrombosis and hypertension. Fortunately, these side effects are relatively rare and observed in only $1.5 \%$ of patients receiving this treatment [68].

Cyclosporine inhibits the cellular and humoral immune response by modifying inflammatory reactions by its influence over the $T_{H}$ cell activation, indirectly inhibiting the production of antibodies and macrophage activation. Patients treated with cyclosporine may experience hypertension, hyperlipidemia, chest pain or cardiac arrhythmias. The most severe complication of cyclosporine therapy is renal failure resulting in hypertension, fluid and electrolytes imbalance (hyperkalemia, hypomagnesemia) and imminent congestive $\mathrm{HF}[69,70]$.

Anakinra, a human interleukin-1 receptor antagonist, was first used in to treat autoimmune disease in 2001. According to more recent reports, it also helps reduce myocardial ischemia in acute coronary syndromes. The exact mechanism of action is not fully known and requires further evaluation [71].

Summary of the CV side effects of the drugs used in AD therapy is shown in Table 2.

Implantable cardioverter-defibrillators have proven themselves in preventing sudden cardiac death, in cardiac patients, however there is lacking data on their use in patients who manifest life threatening arrhythmias as a result of autoimmune disease and therefore a further investigation is required.

\section{Conclusions}

Multisystem involvement is the hallmark of $\mathrm{AD}$, and the $\mathrm{CV}$ system is a frequent target of the most common systemic autoimmune diseases. In the last several decades, the increased prevalence of $\mathrm{CV}$ complications in patients with $\mathrm{AD}$ has been observed. CV manifestations range from mild 
Table 2. Side effect of drugs used in autoimmune diseases of the cardiovascular system.

\begin{tabular}{|c|c|}
\hline Drug & Side effects from the cardiovascular system \\
\hline Glicocorticosteroids & $\begin{array}{l}\text { Arterial hypertension, diabetes, thromboembolic complications, renal impairment, } \\
\text { heart failure }\end{array}$ \\
\hline $\begin{array}{l}\text { Nonsteroidal anti- } \\
\text { inflammatory drugs }\end{array}$ & Edema, arterial hypertension, worsening heart failure, acute coronary events \\
\hline Sulfasalazine & Pericarditis \\
\hline Leflunomid & Arterial hypertension \\
\hline Chloroquine & $\begin{array}{l}\text { Arterial hypotension, changes in the ECG: ORS prolongation, QT prolongation, } \\
\text { T wave changes, cardiomyopathy, III degree atrioventricular block, heart failure, } \\
\text { ventricular arrhythmias }\end{array}$ \\
\hline Infliximab & Arrhythmias, arterial hypertension, heart failure aggravation, arterial hypotension \\
\hline Etanercept & $\begin{array}{l}\text { Coronary artery disease, myocardial infarction, heart failure, thrombosis, arterial } \\
\text { hypertension }\end{array}$ \\
\hline Cyclosporine & Chest pain, irregular heartbeat, kidney failure, arterial hypertension, heart failure \\
\hline \multirow[t]{2}{*}{ Metotrexate } & $\begin{array}{l}\text { Pericardial effusion, pulmonary fibrosis, asthma, pulmonary hypertension, } \\
\text { heart failure, diabetes }\end{array}$ \\
\hline & Beneficial effect of reducing atherosclerotic lesions in coronary arteries \\
\hline Anakinra & Beneficial effect of reducing the area of ischemia in acute coronary syndromes \\
\hline
\end{tabular}

to severe, even being the initial presentation of AD. Cardiologists should remain vigilant to for underlying diseases. Since silent CV involvement is common, it is rarely recognized.

Both autoimmune diseases and their management have a significant impact on the CV system, which results in decreased quality of life, higher mortality and increased cost of healthcare. Improving the quality of life, preventing CV adverse events, decreasing hospitalizations and mortality, all-the-while minimizing pharmacological side effects is the present goal of this multidisciplinary management of autoimmune disease.

\section{Acknowledgements}

This work is a part of the project 'Predicting adverse clinical outcomes in patients with implanted defibrillating devices', which was supported by the Foundation for Polish Science - TEAM program co-financed by the European Union within the European Regional Development Fund.

Conflict of interest: none declared

\section{References}

1. Bonow RO, Mann DL, Zipes DP et al. Braunwald's heart disease: A textbook of cardiovascular medicine. Saunders, Philadelphia 2011: $2107-2116$.

2. Knockaert DC. Cardiac involvement in systemic inflammatory diseases. Eur Heart J, 2007; 28: 1797-1804.

3. Villa-Forte A, Mandell BF. Cardiovascular disorders and rheumatic disease. Rev Esp Cardiol, 2011; 64: 809-817.

4. Kitas G, Banks MJ, Bacon PB. Cardiac involvement in rheumatoid disease. Clin Med, 2001; 1: 18-21.
5. Crozier IG, Li E, Milne MJ, Nicholls MG. Cardiac involvement in systemic lupus erythematosus detected by echocardiography. Am J Cardiol, 1990; 65: 1145-1148.

6. Doria A, Iaccarino L, Sarzi-Puttini P, Atzeni F, Turriel M, Petri M. Cardiac involvement in systemic lupus erythematosus. Lupus, 2005; 14: 683-686.

7. Rosenbaum E, Krebs E, Cohen $\mathrm{M}$ et al. The spectrum of clinical manifestations, outcome and treatment of pericardial tamponade in patients with systemic lupus erythematosus: a retrospective study and literature review. Lupus, 2009; 18: 608-612.

8. Nihoyannopoulos P, Gomez PM, Joshi J et al. Cardiac abnormalities in systemic lupus erythematosus. Association with raised anticardiolipin antibodies. Circulation, 1990; 82: 369-375.

9. Fernández-Dueñas J, López-Granados A. Severe mitral regurgitation in libman-sacks endocarditis conservative surgery. Rev Esp Cardiol, 2005; 58: 1118-1120.

10. Moaref AR, Afifi S, Rezajan S, Rezajan GR. Isolated tricuspid valve libman-sacks endocarditis and valvular stenosis: Unusual manifestations of systemic lupus erythematosus. J Am Society Echocardiog, 2010; 23 : 341-345.

11. Moder KG, Miller TD, Tazelaar HD. Cardiac involvement in systemic lupus erythematosus. Mayo Clin Proc, 1999; 74: 275-284.

12. Moyssakis I, Tekonidou M, Vassilios V et al. Libman-Sacks endocarditis in systemic lupus erythematosus: Prevalence, associations, and evolution. Am J Med, 2007; 120: 636-642.

13. Van der Laan-Baalbergen NE, Mollema SA, Kritikos H. Heart failure as presenting manifestation of cardiac involvement in systemic lupus erythematosus. Neth J Med, 2009; 67: 295-301.

14. Ohara N, Myiata T, Kurata A, Oshiro H, Sato O, Shigematsu H. Ten years' experience of aortic aneurysm associated with systemic lupus erythematosus. Eur J Vasc Endovasc Surg, 2000; 19: 288-293.

15. Roldan CA, Shively BK, Crawford MH. An echocardiographic study of valvular heart disease associated with systemic lupus erythematosus. N Engl J Med, 1996; 335: 1424-1430.

16. Johnson SR, Gladman DD, Urowitz MB, Ibanez D, Granton JT. Pulmonary hypertension in systemic lupus erythematosus. Lupus, 2004; 13: 506-509.

17. Jordan JM, Valenstein P, Kredich DW. Systemic lupus erythematosus with Libman-Sachs Endocarditis in a 9-month-old infant with neonatal lupus erythematosus and congenital heart block, Pediatrics,1989; 84: 574-578.

18. Urowitz MB, Gladman D, Ibanez D. Atherosclerotic vascular events in a multinational inception cohort of systemic lupus erythematosus, arthritis. Care Res, 2010; 62: 881-887. 
19. Asherson RA, Cervera R. Antiphospholipid antibodies and the heart. Lessons and pitfalls for the cardiologist. Circulation, 1991; 84: 920-923.

20. Bili A, Moss AF, Francis CW et al. Anticardiolipin antibodies and reccurent coronary events: a prospective study of 1150 patients. Circulation, 2000; 102: 1258-1263.

21. Greco T, Conti-Kelly AM, Doyle R et al. Newer antiphospholipid antibodies predict adverse outcomes in patients with acute coronary syndrome. Am J Clin Pathol, 2009; 132: 613-620.

22. Gurlek A, Ozdol C, Pamir G et al. Association between anticardiolipin antibodies and recurrent cardiac events in patients with acute coronary syndrome. Int Heart J, 2005; 46: 631-638.

23. Vaarala $\mathrm{O}$, Mänttäri $\mathrm{M}$, Manninen $\mathrm{V}$ et al. Anticardiolipin antibodies and risk of myocardial infarction in a prospective cohort of middle-aged men. Circulation, 1995; 91: 23-27.

24. Koniari I, Siminelaki SN, Baikoussis GN et al. Antiphospholipid syndrome; its implication in cardiovascular diseases: A review. J Cardiothorac Surg, 2010; 5: 101.

25. Turiel M, Muzzupappa S, Gottardi B. Evaluation of cardiac abnormalities and embolic sources in primary antiphospholipid syndrome by transesophageal echocardiography. Lupus, 2000;9:406-412.

26. Hojnik M, George J, Ziporen L, Shoenfeld Y. Heart valve involvement (Libman-Sacks endocarditis) in the antiphospholipid syndrome. Circulation, 1996;92:1579-1587.

27. Plastiras SC, Toumanidis ST. Systemic sclerosis: The heart of the matter, hellenic. J Cardiol 2012; 53: 287-300.

28. Byers RJ, Marshall DAS, Freemont AJ. Pericardial involvement in systemic sclerosis. Ann Rheum Dis, 1997; 56: 393-394.

29. Nakajima K, Kawano M, Hasegawa M et al. Myocardial damages in systemic sclerosis detected by gated myocardial perfusion SPECT sympathetic imaging. Circ J, 2006; 70: 1481-1487.

30. Vacca A, Siotto P, Cauli A et al. Absence of epicardial coronary stenosis in patients with systemic sclerosis and severe impairment of coronary flow reserve. Ann Rheum Dis, 2006; 65: 274-275.

31. York M, Farber HW. Pulmonary hypertension: Screening and evaluation in scleroderma. Curr Opin Rheumatol, 2011; 23: 536-544.

32. Chang B, Schachna L, White B, Wigley FM, Wise RA. Natural history of mildmoderate pulmonary hypertension and the risk factors for severe pulmonary hypertension in scleroderma. J Rheumatol, 2006; 33: 269.

33. Mathai SC, Hummers LK, Champion H. Survival in pulmonary hypertension associated with the scleroderma spectrum of diseases impact of interstitial lung disease. Arthritis Rheum, 2009; 60: 569-577.

34. Kahan A, Coghlan G, McLaughlin V. Cardiac complications of systemic sclerosis. Rheumatolog, 2009; 48: 45-48.

35. Moyssakis I, Gialafos E, Vassiliou V et al. Aortic stiffness in systemic sclerosis is increased independently of the extent of skin involvement. Rheumatology, 2005; 44: 251-254.

36. Nomeir AM, Turner RA, Watts LE. Cardiac involvement in rheumatoid arthritis: follow-up study. Arthritis Rheum, 1979; 22: 561-564.

37. Nicola P, Crowson CS, Maradit-Kremers $\mathrm{H}$ et al. Contribution of congestive heart failure and ischemic heart disease to excess mortality in rheumatoid arthritis. Arthritis Rheum, 2006; 54: 60-67.

38. Udayakumar N, Venkatesan S, Rajendiran C. Diastolic function abnormalities in rheumatoid arthritis: relation with duration of disease. Singapore Med J, 2007; 48: 537.

39. Chung $\mathrm{CP}$, Oeser $\mathrm{A}$, Raggi $\mathrm{P}$ et al. Increased coronary-artery atherosclerosis in rheumatoid arthritis. Arthritis Rheum, 2005; 52: 3045-3053.

40. Francis ML, Varghese JJ, Mathew JM et al. Outcomes in patients with rheumatoid arthritis and myocardial infarction. Am J Med, 2010; 123: 922-928.

41. Gonzalez-Juanatey C, Llorca J, Testa A, Revuelta J, Garcia-Porrua C, Gonzalez-Gay MA. Increased prevalence of severe subclinical atherosclerotic findings in long-term treated rheumatoid arthritis patients without clinically evident atherosclerotic disease. Medicine, 2003; 82: 407-413.

42. Salmon JE, Roman MJ. Subclinical atherosclerosis in rheumatoid arthritis and systemic lupus erythematosus. Am J Med, 2008; 121: 53-58.

43. Maradit-Kremers H, Crowson CS, Nicola P. Increased unrecognized coronary heart disease and sudden deaths in rheumatoid arthritis a population-based cohort study. Arthritis Rheum, 2005; 52: 402-411.

44. Seyfeli E, Guler H, Akoglu S et al. Right ventricular diastolic abnormalities in rheumatoid arthritis and its relationship with left ventricular and pulmonary involvement. Int J Cardiovasc Imag, 2006; 22: 745-754.

45. Roldan CA, DeLong C, Qualls C, Crawford MH. Characterization of valvular heart disease in rheumatoid arthritis by transesophageal echocardiography and clinical correlates. Am J Cardiol, 2007; 100: 496-502.
46. Choi HK, Hernan MA, Seeger JD, Robins JM, Wolfe F. Methotrexate and mortality in patients with rheumatoid arthritis: a prospective study. Lancet, $2002 ; 359: 1173-1177$.

47. Voskuyl AE. The heart and cardiovascular manifestations in rheumatoid arthritis. Rheumatology, 2006; 45: 4-7.

48. Kremers HM, Crowson CS, Therneau TM et al. High ten-year risk of cardiovascular disease in newly diagnosed rheumatoid arthritis patients: A population-based cohort study. Arthritis Rheumatism, 2008; 58: 2268-2274.

49. Lundbegr IE. The heart in dermatomyositis and polymyositis. Rheumatology, 2006; 45: 18-21.

50. Linos E, Fiorentino D, Lingala B. Atherosclerotic cardiovascular disease and ermatomyositis: an analysis of the Nationwide Inpatient Sample survey. Arthritis Res Therapy, 2013; 15: 1-5.

51. Bergfeldt L. HLAB27-associated cardiac disease. Ann Intern Med, 1997; 127: 621-629.

52. Roldan CA, Chavez J, Wiest PW, Qualls CR, Crawford MH. Aortic root disease associated with ankylosing spondylitis. Am Coll Cardiol, 1998; 32: 1397-1404.

53. Brunner F, Kunz A, Weber U, Kissling R. Ankylosing spondylitis and heart abnormalities: Do cardiac conduction disorders, valve regurgitation and diastolic dysfunction occur more often in male patients with diagnosed ankylosing spondylitis for over 15 years than in the normal population? Clin Rheumatol, 2006; 25: 24-29.

54. Fardet L. Corticosteroid-induced clinical adverse events: Frequency, risk factors and patient's opinion. Br J Dermato, 2007; 157: 142-148.

55. Nakajima A, Doki K, Homma M et al. Investigation of glucocorticoidinduced side effects in patients with autoimmune diseases. Yakugaku Zasshi, 2009; 129: 445-450.

56. Naranjo A, Sokka T, Dezcalzo MA et al. Cardiovascular disease in patients with rheumatoid arthritis: results from the QUEST-RA study. Arthritis Res Ther, 2008; 10: 1-10.

57. Tisseverasinghe A, Bernatsky S, Pineau C. Arterial events in persons with dermatomyositis and polymyositis. J Rheumatol, 2009; 36: 1-4.

58. Batlouni M. Nonsteroidal anti-inflammatory drugs: cardiovascular, cerebrovascular and renal effects. Arq Bras Cardiol, 2010; 94: 556$-563$.

59. Gislason GH, Rasmussen JN, Abildstrom SZ, Schramm TK, Hansen ML. Increased mortality and cardiovascular morbidity associated with use of nonsteroidal anti-inflammatory drugs in chronic heart failure. Arch Intern Med, 2009; 169: 141-149.

60. Zhao SZ, Burke T. Cost of heart failure among hypertensive users of nonspecific NSAIDs and COX-2-specific inhibitors. Am J Manag Care, 2002; 8: 414-427.

61. Costedoat-Chalumeau N, Hulot JS. Heart conduction disorders related to antimalarials toxicity: an analysis of electrocardiograms in 85 patients treated with hydroxychloroquine for connective tissue diseases. Rheumatology, 2007; 46: 808-810.

62. Nurmohamed MT, van Halm VP, Dijkmans BA. Cardiovascular risk profile of antirheumatic agents in patients with osteoarthritis and rheumatoid arthritis. Drugs, 2002; 62: 1599-1609.

63. Guedira N, Hajjaj-Hassouni N, Srairi JE et al. Third-degree atrioventricular block in a patient under chloroquine therapy. Rev Rhum Engl Ed, 1998; 65: 58-62.

64. Veinot JP, Mai KT, Zarychanski R. Chloroquine related cardiac toxicity. J Rheumatol, 1998; 25: 1221-1225.

65. Costedoat-Chalumeau N, Hulot JS, Amoura Z. Cardiomyopathy related to antimalarial therapy with illustrative case report. Cardiology, 2007; 107: 73-80.

66. Fragasso G, Sanvito F, Baratto F. Cardiotoxicity after low-dose chloroquine antimalarial therapy. Heart Vess, 2009; 24: 385-387.

67. Salliot C, van der Heijde D. Long-term safety of methotrexate monotherapy in patients with rheumatoid arthritis: A systematic literature research. Ann Rheum Dis, 2009; 68: 1100-1104.

68. Curtis JR, Kramer J, Martin M et al. Heart failure among younger rheumatoid arthritis and Crohn's patients exposed to TNF- $\alpha$ antagonists. Rheumatology, 2007; 46: 1688-1693.

69. Piot $\mathrm{Ch}$, Croisille $\mathrm{P}$, Staat $\mathrm{P}$. Effect of cyclosporine on reperfusion injury in acute myocardial infarction. N Engl J Med, 2008; 359: 473-481.

70. Mewton N, Croisille P, Gahide G et al. Effect of cyclosporine on left ventricular remodeling after reperfused myocardial infarction. J Am Coll Cardiol, 2010; 55: 1200-1205.

71. Grizzard JD, Biondi-Zoccai G. Interleukin-1 blockade with anakinra to prevent adverse cardiac remodeling after acute myocardial infarction (Virginia Commonwealth University Anakinra Remodeling Trial). Am J Cardiol, 2010; 105: 1371-1377. 